\title{
Conséquences de l'enfoncement du lit de l'Arve sur les berges et les ouvrages Bilan général des transports solides sur le cours d'eau
}

\author{
X. Blanc, F. Pinteur \\ DDE Haute-Savoie
}

\section{Conséquences de l'enfoncement du lit de l'Arve}

La Direction Départementale de l'Equipement de la Haute-Savoie, à la fois gestionnaire du cours d'eau, concepteur et maître d'œuvre de la plupart des grands travaux de la vallée, a été amenée à constater et à prendre en charge techniquement les effets de l'abaissement de l'Arve dans son lit, notamment pour ce qui concerne les ouvrages routiers de franchissement. Elle a donc été à même d'apprécier à la fois les causes et les effets de cet abaissement tant du point de vue technique que du point de vue financier.

L'accroissement constant des besoins en matériaux pour la réalisation des grands projets autoroutiers et routiers ainsi que des programmes de construction de bâtiments, depuis les années 50 jusqu'au début des années 80 , ont conduit à extraire de plus en plus de graviers dans l'Arve tant dans son lit mineur que dans le lit majeur. Ce sont environ 10 millions de $\mathrm{m}^{3}$ qui ont été prélevés dans le lit mineur sur cette période et la DDE est confrontée en permanence aux problèmes induits par cet abaissement : affouillement des fondations des piles et culées de pont, érosion des berges, déstabilisation des digues, etc... Il est possible de dresser un tableau rapide de l'évolution du profil en long de l'Arve durant les 30 dernières années et des répercussions de cette évolution sur la pérennité des ouvrages implantés dans son lit ou sur ses bords.

\author{
Mme Th. Sanchis \\ SRAE Rhône-Alpes
}

\subsection{Evolution du profil en long de l'Arve. Conséquences sur les ouvrages}

Le relevé du profil en long de ligne d'eau réalisé, au cours des années 1912-1913, par le Service des Grandes Forces Hydrauliques permet d'en mesurer l'évolution, depuis cette date, section par section.

Source - Barrage des Houches $(23 \mathrm{~km})$ : En partant de la source, on constate peu d'évolution dans cette section limitée en aval par le barrage des Houches, où apparaît même une tendance à l'exhaussement du lit. Les ouvrages en place sont destinés essentiellement à la protection des berges contre l'érosion et les inondations.

Barrage des Houches - Pont de la Carabotte $(14 \mathrm{~km})$ : Les premiers effets des extractions sont apparus, dans ce secteur, vers les années 1955-1956 et n'ont cessé de s'intensifier jusqu'à la réalisation des ouvrages de stabilisation. La ligne d'eau de 1979 fait apparaître une chute de $10 \mathrm{~m}$ par rapport à 1912 à l'aval des Ponts du Fayet (SNCF et CD 43). A la même date, cette chute est moins importante au niveau du Pont de la Carabotte (CD 39), de l'ordre de $2 \mathrm{~m}$. Cet encaissement a provoqué des affouillements sous les ponts du CD 43 et de la SNCF, rendant indispensables les interventions suivantes : 1974 consolidation des appuis du pont SNCF ( $0,8 \mathrm{MF})-1975$ construction d'un seuil en enrochements à l'aval des ponts SNCF et CD 43 $(\mathrm{h}=7 \mathrm{~m})(9,7 \mathrm{MF})-1977$ protection des culées du pont sur le CD 339 (0,7 MF).

\section{Consequences of the sinking of Arve river on its banks and on bridges and other works \\ Comprehensive study of the solid flow along its whole course}

In the first part, the phenomenon of the sinking of the torrent bed is analysed: its scale, it consequences on banks, bridges, walls and other works, and the solutions adopted in order to stop the process are reviewed. These problems are treated from the point of view of the supervising agency. The technical and financial difficulties usually encountered are described, as well as the means of action available. The second part of the paper is presented in the form of a statement of the quantities of materials entering and leaving the system of reference forming the torrent : flowed and extracted materials, sinking of the bed, trapping of deposits by Verbois reservoir. Contrary to an analytical process, this approach frees the authors from studying the complex mechanisms of Erosion, Transportation and Depositing and allows them to focus on their results. 
Pont de la Carabotte - Cluses $(21 \mathrm{~km})$ : L'enfoncement du lit s'est limité à $2 \mathrm{~m}$ à l'amont de Sallanches, dans la zone endiguée depuis l'époque sarde. La réalisation, de 1961 à 1963, d'un endiguement au droit de la zone industrielle, rive gauche, représente un montant de $15 \mathrm{MF}$, en partie imputable à l'abaissement du lit. L'Arve, à l'aval de Sallanches, a été complètement remodelé par la réalisation de l'Autoroute Blanche.

Cluses - Pont d'Anterne $(7 \mathrm{~km})$ : Les ouvrages de franchissement dans Cluses ont subi les effets des extractions massives réalisées à l'aval. Les derniers levés de ligne d'eau, en 1981, font apparaître des abaissements du lit de $9,5 \mathrm{~m}$ à l'aval du seuil de la Sardagne, $7,6 \mathrm{~m}$ à l'aval de Pressy a Thyez (restitution EDF), pour diminuer graduellement jusqu'au Pont d'Anterne où l'abaissement est de $3 \mathrm{~m}$.

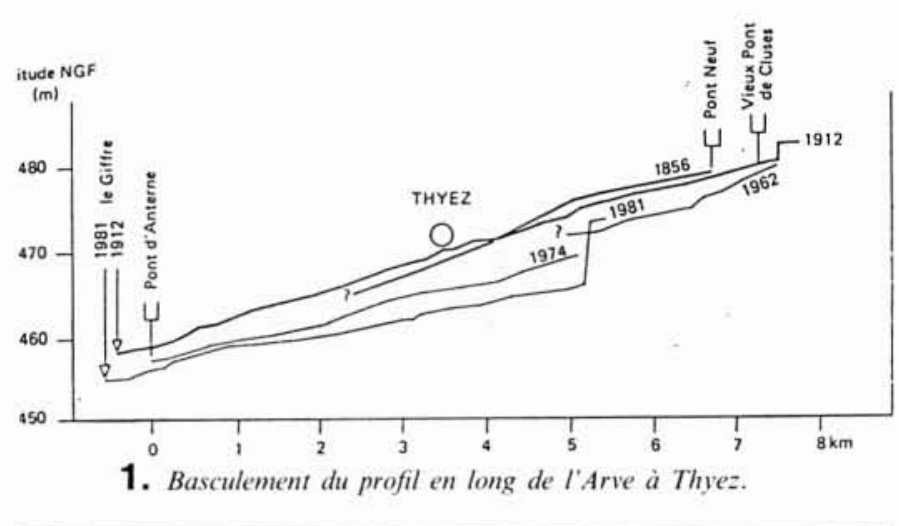

Ils ont conduit à la réalisation d'un seuil en enrochements à l'aval du Pont de la Sardagne en 1963 (3 MF) consolidé en 1984 suite aux dégradations de la crue de novembre 1983 (5,3 MF). Un autre seuil en enrochements, à l'aval de la restitution EDF de Pressy a été réalisé en 1984 (6,1 MF). La consolidation des endiguements minés par l'érosion régressive dans la traversée de Cluses a nécessité l'engagement de 2,7 MF de 1982 à 1984.

Pont d'Anterne - Pont de Bellecombe $(19 \mathrm{~km})$ : L'examen des lignes d'eau, à l'amont de la confluence du Giffre, faitressortir un abaissement de $3,8 \mathrm{~m}$ entre 1912 et 1982 . La reprise en sous-œuvre des appuis du Pont d'Anterne affouillé remonte aux années 1975-1976. En 1983-1984 un radier en enrochements a été posé sous l'ouvrage et prolongé par un seuil complet en 1986 (6,4 MF). L'enfoncement du lit, dans la section endiguée à l'amont du pont SNCF de Bonneville, est de l'ordre de $2 \mathrm{~m}$. Il est au droit du pont de Bonneville de l'ordre de $3 \mathrm{~m}$ par rapport à 1913, atteint localement $4 \mathrm{~m}$, et se réduit progressivement jusqu'à devenir nul au Pont de Bellecombe. En 1974-1975, reprise des appuis du Pont de Bonneville (1,7 MF). En 1977. la réfection de la digue soumise à l'érosion régressive, à la confluence Arve-Borne (2,4 MF), et son prolongement vers l'amont en $1980(0,7 \mathrm{MF})$, ont précédé la réalisation en 1984 de deux seuils en enrochements sous le Pont de Bonneville $(1,2 \mathrm{MF})$ et à l'aval de la confluence ArveBorne (2,0 MF).

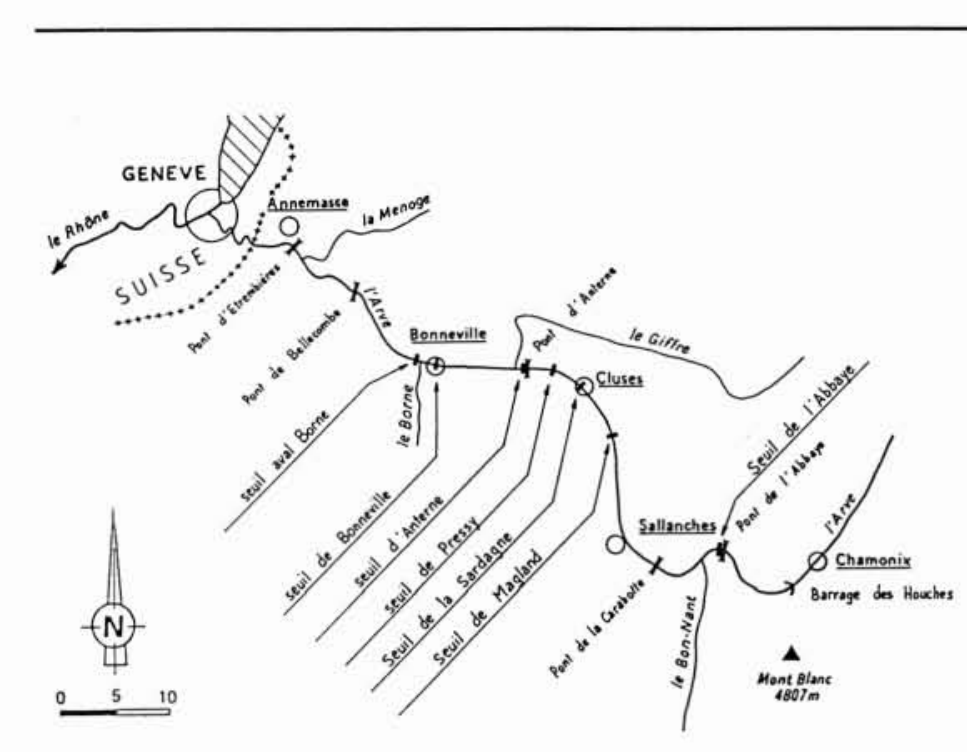

2. Ouvrages de stabilisation.

Pont de Bellecombe - Frontière suisse $(16 \mathrm{~km})$ : Du Pont de Bellecombe au Barrage EDF d'Arthaz, le lit de l'Arve est assez stable, avec tendance à l'exhaussement. De la Ménoge à la Frontière Suisse, les lignes d'eau comparées (1912 et 1987) révèlent un enfoncement du lit inférieur à $2 \mathrm{~m}$ à l'amont du pont routier d'Etrembières RN 205, et de l'ordre de $4 \mathrm{~m}$ à l'aval, conséquence directe des extractions de matériaux, essentiellement localisées à l'aval des ponts d'Etrembières. L'ensemble des grands travaux de protection des berges et des ouvrages reste à faire (seuils, endiguements, recalibrage du lit) et sont estimés à $30 \mathrm{MF}$.

\subsection{Incidence de l'évolution du lit de l'Arve sur les projets routiers}

La prise en compte des phénomènes précédemment décrits dans la conception et l'économie des grands projets a conduit les maîtres d'ouvrage à faire établir les études hydrauliques définissant les ouvrages aptes à garantir la pérennité des infrastructures routières. Ces études et ces travaux ont concerné essentiellement la construction de l'Autoroute Blanche et celle de la voie express Le FayetLes Houches.

Autoroute Blanche: La contiguïté de l'Arve et de l'autoroute a conduit à la réalisation de travaux d'aménagement du lit : Endiguement à l'amont de Sallanches sur plus d'1 km, en 1975 (12 MF) - Cinq rectifications au lit et protections de 3 ponts à Magland en 1975 (18,1 MF) Seuil à l'amont de Cluses en 1974 (1,2 MF) - Rectification d'Anterne en 1973 (3,3 MF) - Rectification de Vougy en 1970-1973 (5 MF) - Rectification d'Etrembières et protection du pont (4,5 MF). Soit un coût total des ouvrages de protection contre l'Arve de 44,1 MF. A ces investissements, il faut ajouter les travaux d'entretien et la réalisa- 
tion de seuils, ou d'autres protections, compensant les effets d'érosion du lit depuis sa construction, soit $8,7 \mathrm{MF}$ étalés sur 10 ans.

Voie express Le Fayet - Les Houches : Pour achever la liaison Autoroute-tunnel du Mont-Blanc, la construction de la route express par mise à $2 \times 2$ voies de l'ancien itinéraire RN 205 a nécessité des travaux gigantesques (viaduc des Egrats $2,2 \mathrm{~km}$ de long et tunnels $2,4 \mathrm{~km}$ ) qu'il convenait de protéger de l'Arve. Les travaux engagés représentent un surcoût important (19,2 MF) : Protections latérales à l'aval du barrage des Houches (4 MF) Protection du Viaduc des Egrats (10,5 MF) - Protection de la voie descendante RN 206 à Passy (3,2 MF) Compléments de protection à prévoir 1988-1989 (1,5 MF).

\subsection{Conclusion}

Le montant global des investissements réalisés pour se protéger des attaques de l'Arve, fortement aggravées par les extractions de matériaux dans le lit mineur, ressort en valeur actuelle à $126 \mathrm{MF}$. A ces sommes dépensées il faut ajouter l'estimation des investissements à prévoir pour réaliser les travaux indispensables au maintien de la situation actuelle soit environ $60 \mathrm{MF}$. Un rapprochement de ces chiffres avec les volumes extraits en lit mineur reste simpliste mais situe cependant l'incidence financière des conséquences des extractions de matériaux dans l'Arve.

\section{Bilan des transports solides de l'Arve}

L'enfoncement du lit précédemment décrit traduit le net déséquilibre entre les matériaux apportés à l'Arve (par ses affluents principalement) et les matériaux exportés (par le cours d'eau lui-même en direction du Rhône, ou par le biais des extractions de matériaux). La collecte entreprise par le groupe de travail "Arve " de l'ensemble des informations existant sur le phénomène a permis d'évaluer les quantités de matériaux mises en jeu par ces mouvements, et ainsi de dresser une esquisse de bilan des transports solides du cours d'eau.

\subsection{Méthodologie d'approche}

Enoncé du bilan: Sur un système de référence donné, on peut écrire la relation :

$$
E=S+P-\Delta \text {, dans laquelle }
$$

$E$ représente les matériaux entrants dans le système (apport de matériaux amont, ou latéral par les torrents et les rivières affluentes)

$S$ représente les matériaux sortant du système par voie hydraulique (charriage et transport en suspension)

$P$ représente les matériaux prélevés au système par extractions et curages

$\Delta$ traduit la variation du stock de matériaux en place (si $\Delta=0$ stabilité du lit : les apports au système équilibrent les exports - le lit est stable ; si $\Delta>0$ déstocka- ge : les apports ne permettent pas de compenser les exports - ceux-ci se font au détriment des réserves du lit, qui s'enfonce ; si $\Delta<0$ engraissement : les apports sont plus importants que les exports; les conditions sont réunies pour que la rivière constitue ses propres réserves, ce qui se traduit par un exhaussement du lit).

Définition du système de référence: On prendra comme système de référence : l'ensemble du cours de l'Arve dans sa plaine alluviale, et la période 1950-1985.

Le choix de la période résulte de la conjonction de plusieurs constatations : ampleur des prélèvements depuis 1950 - absence d'enfoncement significatif du lit jusqu'en 1955 - piégeage des matériaux exportés au Rhône par la retenue de Verbois depuis 1944, et dont le remplissage est régulièrement suivi.

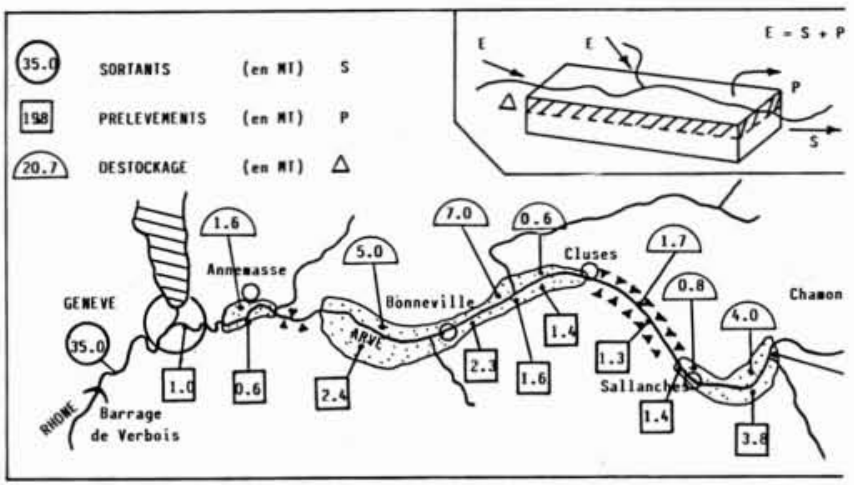

3. Détail des matériaux sortants, prélevés et déstockès sur l'Arve pendant la période 1950-1985 (en $\mathrm{Mt}$ ).

\subsection{Les termes du bilan}

Les entrants $E$ : Les entrants dans le système sont constitués par les apports de l'Arve amont, de caractère fortement torrentiel, des torrents, et des rivières affluentes, éventuellement par les apports directs dus aux avalanches et aux éboulements.

Le caractère sporadique de ces transports, la diversité des régimes torrentiels (apports glaciaires, laves torrentielles, décapages de versants, ...) interdisent toute estimation chiffrée directe de ce terme.

Les sortants $S$ : Les matériaux exportés par l'Arve vers le Rhône peuvent être estimés par deux approches:

- des mesures hebdomadaires sur L'Arve à Genève (annuaire hydrologique Suisse), disponibles sur la période 1965-1983, qui font état d'une moyenne de 1 Mt de matériaux par an transportés en suspension ;

- le remplissage de la retenue de Verbois, qui s'étend du confluent de l'Arve et du Rhône, jusqu'au barrage luimême, situé à $12 \mathrm{~km}$ à l'aval, dans la mesure où l'on peut considérer que les sédiments ne sont pas issus du Rhône, compte tenu du rôle de décanteur joué par le lac Léman. L'important travail de relevés bathymétriques effectué par le Service de l'électricité de Genève, gestionnaire de l'ou- 
vrage, permet une approche relativement précise de la sédimentation annuelle moyenne dans la retenue sur la même période, de $400000 \mathrm{~m}^{3}$, soit de l'ordre de $560000 \mathrm{t}$.

On voit qu’à peine plus de la moitié des matériaux transportés en suspension au niveau de Genève seraient sédimentés dans la retenue de Verbois, le reste transitant vraisemblablement au travers du barrage en période d'exploitation normale. On ne dispose d'aucune donnée sur le transport par charriage, mais les mesures granulométriques sur la retenue de Verbois donnent certaines indications: même en queue de retenue le percentile le plus grossier reste inférieur à 400 microns ; de plus de D 95 reste inférieur à 200 microns sur les trois quarts aval de la retenue, là où s'accumulent la quasi-totalité des matériaux sédimentés; ceci incite à penser que ce transport par charriage est négligeable vis-à-vis du bilan global ; ce point semble corroboré par l'arrêt quasi-total des extractions à Genève, qui ne sortaient plus que « des sables fins et des limons".

Il a été pris comme hypothèse que les matériaux sédimentés dans Verbois ont une densité sèche de 1,4.

Les prélèvements $P$ : La reconstitution historique des extractions de matériaux et curages a permis d'évaluer les quantités de matériaux prélevés à l'Arve, représentés dans la figure 3 .

Ces matériaux sont prélevés dans le lit, et subissent donc un lavage partiel. Les courbes granulométriques de contrôle font apparaître que globalement plus de $90 \%$ des tonnages extraits sont $>200$ microns. On peut estimer que leur densité sèche est de 1,6.

Le déstockage $\Delta$ : La comparaison des profils en long de 1912 (que l'on peut considérer comme représentatifs de l'état jusqu'en 1955), et de profils établis pendant la période de référence, permet de mesurer l'ampleur des enfoncements connus par la rivière.

Traduire ces enfoncements en terme de volumes déstockés, en considérant (faute de profils en travers) que ces enfoncements ont eu lieu de manière homogène sur la totalité du lit mineur, sans affecter le lit majeur, constitue une démarche certainement critiquable à maints égards. Néanmoins, les études faites sur le sujet montrent que la brutalité de l'incision a fait perdre à la rivière ses caractéristiques de cours d'eau tressé, et les profils en travers dressés récemment font apparaître un chenal étroit et profond, de section relativement géométrique, même dans les tronçons non endiguées. Sous ces réserves, les volumes déstockés ont pu être évalués, et sont représentés sur la figure 3 .

Les matériaux en place possèdent une granulométrie évidemment hétérogène, mais on peut penser que les fines (200 microns) représentent moins de $30 \%$ des alluvions. Leur densité sèche a été estimée à 1,8 .

\subsection{Bilan des transports solides}

Dans la relation : $E=S+P-\Delta$, on a pu estimer 3 des 4 termes, et la valeur des apports à l'Arve peut donc en être déduite. Néanmoins, et compte tenu de l'imprécision liée à l'évaluation de chacun de ces termes, le tableau cidessous situe d'avantage un ordre de grandeur des phénomènes en cause qu'il ne représente un réel chiffrage.

Tableau récapitulatif des entrées/sorties de matériaux dans l'Arve sur la période 1950-1985 (en Mt)

\begin{tabular}{|c|r|r|l|l|l|l|}
\hline & \multicolumn{3}{|c|}{$\begin{array}{c}\text { Tonnage total } \\
\text { (en Mt) }\end{array}$} & \multicolumn{3}{c|}{$\begin{array}{c}\text { Tonnage moyen } \\
\text { annuel (en Mt) }\end{array}$} \\
\hline $\begin{array}{l}\text { Classe } \\
\text { granulo }\end{array}$ & $200 \mu$ & $200 \mu$ & Total & $200 \mu$ & $200 \mu$ & Total \\
\hline $\begin{array}{c}\text { Termes } \\
\text { du bilan }\end{array}$ & & & & & & \\
$S$ & 31,5 & 3,5 & 35,0 & 0,9 & 0,1 & 1,0 \\
$P$ & 2,5 & 17,5 & 20,0 & 0,1 & 0,5 & 0,6 \\
$\Delta$ & 6,0 & 15,0 & 21,0 & 0,2 & 0,4 & 0,6 \\
$E$ & 28,0 & 6,0 & 34,0 & 0,8 & 0,2 & 1,0 \\
\hline
\end{tabular}

1) Sur la période étudiée, on constate que les prélèvements sont du même ordre de grandeur que le déstockage, ce qui laisse à penser qu'ils ont été effectués entièrement au détriment des matériaux en place, et non sur un éventuel "débit solide", pourtant une quantité extrêmement importante de matériaux, pendant la même période, a été apportée au Rhône. En fait, si l'on introduit la nature des matériaux considérés, on explique parfaitement cette apparente contradiction : les prélèvements ne concernent quasiment que des matériaux grossiers, et seuls sont apportés au Rhône les matériaux les plus fins.

2) Si le flux de matériaux solides semble très faible pour ce qui concerne les éléments grossiers (en tous cas sans commune mesure avec les niveaux d'extractions atteints), en revanche, les estimations font apparaitre un important flux de fines; ces fines ne proviennent que partiellement de la remise en suspension des matériaux en place lors du déstockage; cette déduction est d'ailleurs confirmée par l'important remplissage de Verbois dès les premières années, alors même que les enfoncements du lit étaient quasiment inexistants. Sans aller trop loin dans l'interprétation de ces estimations, il semble net que la majeure partie des apports des affluents et des torrents soient constituée de fines (de l'ordre des trois quarts).

3) La globalité de cette approche masque le fait qu'il peut parfaitement exister des zones où la compétence du cours d'eau ne permet pas l'entraînement des matériaux les plus grossiers, et où des désordres sont susceptibles de se produire par excès d'engraissement (alors même que le bilan général fait apparaître un déficit). Ces cas peuvent soit avoir des causes naturelles (ex. : confluence d'un torrent glaciaire qui apporte de très gros éléments), soit être le résultat d'actions anthropiques (ex. du barrage des Houches, qui bloque les matériaux, sans que les chasses effectuées soient à même d'entraîner les plus grossiers, et où par conséquent les enlèvements représentent une nécessité). 
4) L'ensemble de ces réflexions permet de renforcer la politique de gestion actuellement menée en HauteSavoie :

- privilégier la notion de curage (dans l'intérêt du cours d'eau) à la notion d'extractions (dans l'intérêt de l'extracteur !), en déterminant les volumes à prélever sur la base d'une connaissance globale de la dynamique du cours d'eau ;

- rechercher pour les zones d'engraissement local, chaque fois que cela est possible, des solutions techniques visant à améliorer l'hydraulicité et la compétence du cours d'eau, pour éviter des curages dont les conséquences demeurent en tout état de cause aléatoires.

\section{Bibliographie}

BCEOM (déc. 1987), Etude d'aménagement hydraulique de l'Arve entre la frontière suisse et le pont de Bellecombe, $60 \mathrm{p} .+$ annexes et plans.

COHEN M., J. BRuschin (1985), Les chasses de la retenue de Verbois (1945-1984). Services Industriels de Genève; Ecole Polytechnique Fédérale de Lausanne, laboratoire d'hydraulique, 54 pages.

PeIRY J. L. (janv. 1988), Approche géographique de la dynamique spatio-temporelle des sédiments d'un cours d'eau intramontagnard. L'exemple de la plaine alluviale de l'Arve (HauteSavoie), 378 p.

SOGREAH (de 1971 à 1985), Etudes hydrauliques sur l'ensemble du cours de l'Arve. 